AperTO - Archivio Istituzionale Open Access dell'Università di Torino

\title{
The EUSO-Balloon pathfinder
}

\section{This is a pre print version of the following article:}

Original Citation:

Availability:

This version is available http://hdl.handle.net/2318/1578468

since 2016-06-30T15:22:07Z

Published version:

DOI:10.1007/s10686-015-9467-9

Terms of use:

Open Access

Anyone can freely access the full text of works made available as "Open Access". Works made available under a Creative Commons license can be used according to the terms and conditions of said license. Use of all other works requires consent of the right holder (author or publisher) if not exempted from copyright protection by the applicable law. 


\title{
The EUSO-Balloon pathfinder
}

\author{
The JEM-EUSO Collaboration
}

Received: date / Accepted: date

\begin{abstract}
EUSO-Balloon is a pathfinder for JEM-EUSO, the Extreme Universe Space Observatory which is to be hosted on-board the International Space Station. As JEM-EUSO is designed to observe Ultra-High Energy Cosmic Rays (UHECR)-induced Extensive Air Showers (EAS) by detecting their ultraviolet light tracks "from above", EUSO-Balloon is a nadir-pointing UV telescope too. With its Fresnel Optics and Photo-Detector Module, the instrument monitors a $50 \mathrm{~km}^{2}$ ground surface area in a wavelength band of 290-430 $\mathrm{nm}$, collecting series of images at a rate of 400,000 frames/sec. The objectives of the balloon demonstrator are threefold: a) perform a full end-to-end test of a JEM-EUSO prototype consisting of all the main subsystems of the space experiment, b) measure the effective terrestrial UV background, with a spatial and temporal resolution relevant for JEM-EUSO. c) detect tracks of ultraviolet light from near space for the first time. The latter is a milestone in the development of UHECR science, paving the way for any future space-based UHECR observatory.

On August 25, 2014, EUSO-Balloon was launched from Timmins Stratospheric Balloon Base (Ontario, Canada) by the balloon division of the French Space Agency CNES. From a float altitude of $38 \mathrm{~km}$, the instrument operated during the entire astronomical night, observing UV-light from a variety of groundcovers and from hundreds of simulated EASs, produced by flashers and a laser during a two-hour helicopter under-flight.
\end{abstract}

Keywords UHECR · air-fluorescence · JEM-EUSO · balloon experiment

The JEM-EUSO Collaboration

for the full author list see the last page of this article.

Corresponding author:

Peter von Ballmoos

Institut de Recherche en Astrophysique et Planétologie, Toulouse, France

Tel.: + 33561556647

E-mail: pvb@irap.omp.eu 


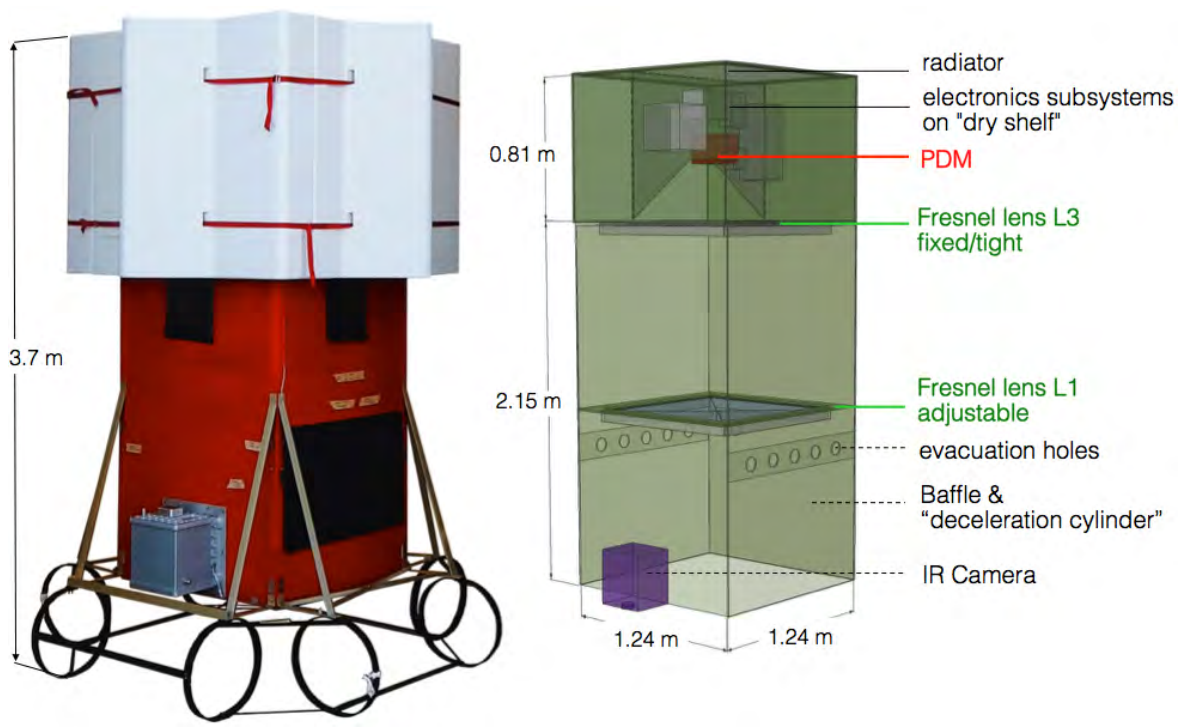

Fig. 1 left: EUSO-Balloon, ready for its first flight, Timmins, Ontario (Ca), August 2014 right: schematic view of the instrument booth and optical bench, without floaters and "crash rings".

\section{Context}

EUSO-Balloon is developed by the JEM-EUSO collaboration as a demonstrator for the key technologies and methods featured in the forthcoming space instrument [1]. JEM-EUSO is designed to observe ultra high-energy cosmic rays (UHECRs) by looking downward to the Earth's atmosphere from the ISS, observing the UV fluorescence light of UHECR-induced Extensive Air Showers (EAS). This volume contains a number of detailed articles on JEMEUSO, notably its status [1], the science case [2], [3], and an overview on the instruments [4].

Whereas the physics and the detection technique of EAS through ultraviolet light (UV) emission is well established and used daily in ground based detectors (see e.g. the Pierre Auger Observatory [5], or the Telescope Array [6]), their observation from space has never been performed. Thorough knowledge of the earth's UV background, and particularly its spatial and temporal variability, is an important prerequisite for the JEM-EUSO mission. Like JEM-EUSO, EUSO-Balloon is a nadir-pointing UV telescope, monitoring the atmosphere from above. Although observing from a float altitude of "only" $\sim 40 \mathrm{~km}$, EUSO-Balloon performs a full scale end-to-end test of all the key technologies and instrumentation for the JEM-EUSO mission - as well as any future mission dedicated to the observation of extensive air showers from space.

The EUSO-Balloon mission has been proposed in 2011 to the French Space Agency CNES by the three French laboratories (APC, IRAP and LAL) involved in the international JEM-EUSO collaboration. All relevant institutions 
Table 1 Responsibilities in the procurement and delivery of the various components of the EUSO-Balloon instrument, and the LASER/flasher tests during the Timmins flight. The full names and addresses of the contributing institutions are listed in the end of this article.

\begin{tabular}{llll}
\hline System & Sub-Assembly & Lab / University & Country \\
\hline Telescope & Fresnel Lenses & RIKEN & Japan \\
& Lens Fitting & IRAP & France \\
& Telescope Structure / Gondola & IRAP & France \\
\hline PDM & Multi-Anode Photo-Multipliers & RIKEN & Japan \\
& ASIC & LAL & France \\
& Elementary Cell & LAL, KIT & France, Germany \\
& HV Power Supply and Switches & NCBJ, APC & Poland, France \\
& PDM Board & EWHA & South Korea \\
& PDM Low Power Supply & UNAM & Mexico \\
& PDM Structure & INFN Frascati & Italy \\
\hline Processor & Main processing unit & INFN Napoli & Italy \\
& Data Storage & INFN Napoli & Italy \\
& Clock Generator & INFN Napoli & Italy \\
& Trigger software & INFN Torino & Italy \\
Digital & CCB Data Processor & IAAT & Germany \\
& House Keeping Board & UNAM & Mexico \\
& Low Voltage Power Supply & UNAM & Mexico \\
\hline Battery Pack & Batteries and Controller & UAH, IRAP & Spain,France \\
\hline IR-CAM & IR Camera & UAH & Spain \\
\hline Helicopter & UV-LASER, LED and & UHA, CSM & USA \\
\hline
\end{tabular}

and international partners within the JEM-EUSO collaboration contribute to the instrument (Table 1) according to their corresponding tasks and responsibilities within JEM-EUSO. As a CNES-led project, EUSO-Balloon was kicked off in September 2011 and went through a phase A study, demonstrating its feasibility by the end of the same year. The instrument definition (phase B) was carried out in 2012, with the production and the qualification of crucial subsystems (PDM, DP - see below) being led in parallel by the institutes of the JEM-EUSO collaboration. The manufacturing of all the subsystems was accomplished by the end of 2013, and the instrument was integrated and tested by June 2014 .

On August 25, 2014 EUSO-Balloon has performed a successful maiden flight after a launch by the French Space Agency CNES from the Stratospheric Balloon Base in Timmins, Ontario (Ca). The present instrumental paper exposes the objectives of EUSO-Balloon (section 2); it describes the instrument and its subsystems (section 3), summarizes the balloon flight of August 2014 (section 4), and concludes with the perspectives of planned future balloon flights (section 5). The 2014 balloon flight produced a multitude of results that will be reported in detail in the proceedings of the $34^{\text {th }}$ ICRC $[7][8][9][10][11][12][13][14][15][16]$. 


\section{Objectives}

A) technology demonstrator: Among the crucial technologies that benefit from the balloon flights are the High Voltage (HV) power supplies, the HV switches (HV relays commuting the $\mathrm{HV}$ in case a bright atmospheric event comes into the FOV), the Front-End Electronics (including the ASICs and FPGA), the on-board hardware and software algorithms involved in the triggering and recognition of cosmic-ray initiated air showers.

B) data acquisition and background study: An accurate measurement of the background light / airglow in the near UV region is an important prerequisite for the JEM-EUSO mission. Whereas a number of background measurements have been performed by previous balloon missions [17] [18], and even from space [19], no imaging instrument has been employed so far, resulting in "spatial resolutions" that were extremely modest. Localized background signals were washed out by the integration over a large surface and, likewise, temporal variations on small scales were not observable, and thus went unconstrained. The B) objectives are thus:

- experimental confirmation of the effective background below $40 \mathrm{~km}$ observed with a pixel size on ground representative for JEM-EUSO (150 m x $150 \mathrm{~m}$ in $\mathrm{a} \pm 5.5^{\circ}$ field of view),

- acquisition of UV signal and background in a format similar to JEM-EUSO,

- testing of observational modes and switching algorithms,

- testing/optimizing trigger algorithms with real observations, i.e. different ground-covers and time-variable background,

- testing of the acquisition capability of the infrared-camera (IRcam).

C) pioneering mission for JEM-EUSO: The ultimate objective for EUSOBalloon is the actual detection of EASs by looking downward from the edge of space. Since detecting these obviously rare events is unlikely during a first short balloon flight (threshold $\simeq 10^{18} \mathrm{eV}$ ), LASER-induced events provide a proof of principle and a way to calibrate the sensitivity.

\section{Overview of the $E U S O-B a l l o o n$ instrument}

The general layout of EUSO-Balloon is shown in Fig. 1, its main components are the optical bench and the instrument booth. The main driver for determining the optical specifications - i.e. the pixel size and the field of view - was to observe a representative background within the pixel of a standard JEM-EUSO photodetector module (PDM). The EUSO Simulation and Analysis Framework (ESAF) has been adapted to simulate the response of the instrument (see e.g. $[20]$ ). The configuration used for JEM-EUSO has been modified, scaling for the altitude of the instrument, changing the surface parameterization, introducing the new optical system and field of view (see Table 2). An electronic block diagram of the entire instrument, summarizing all subsystems and components is shown in Fig. 2. The development of all components and sub-assemblies is 
Table 2 Comparison of the principle characteristics between JEM-EUSO and EUSOBalloon. The field of view of EUSO-Balloon - and hence its pixel size - has been dimensioned to see a level of background that is comparable to the one expected for JEM-EUSO.

\begin{tabular}{lll}
\hline & JEM-EUSO & EUSO-Balloon \\
\hline Number of PDMs & 137 & 1 \\
Flight Altitude [km] & 420 & 38 \\
Diameter of Optics [m] & 2.5 & 1 \\
Field of View / PDM & $3.8^{\circ}$ & $11^{\circ}$ \\
PDM@ground [km] & 28.2 & 7.3 \\
Field of View / pixel & $0.08^{\circ}$ & $0.23^{\circ}$ \\
Pixel@ground [km] & 0.580 & 0.150 \\
Signal w/r to JEM-EUSO & 1 & 17.6 \\
BG w/r to JEM-EUSO & 1 & $0.9-1.8$ \\
S/ $\sqrt{\mathrm{N}}$ w/r to JEM-EUSO & 1 & $20-10$ \\
Threshold Energy [eV] & $3 \cdot 10^{19}$ & $1.5-3 \cdot 10^{18}$ \\
\hline
\end{tabular}

based on similar JEM-EUSO components and sub-assemblies, designed and built entirely within the JEM-EUSO collaboration (see Table 2).

Besides the focal plane detector (PDM) and associated electronics (DP) which are described below, the instrument booth houses the telemetry system (SIREN), CNES specific instrumentation (ICDV, Hub), and two batterypacks.

\subsection{The Photo-Detector Module (PDM)}

The UV light collected by the telescope is focused onto - and detected by - the PDM (Fig. 3), which is composed of 36 Multi-Anode Photomultiplier Tubes (MAPMT), associated front-end electronics, High-Voltage Power supplies, and trigger logic. The key characteristics of this UV camera are its spatial resolution of a few $\mathrm{mm}$, its time resolution of a few ns, a quantum efficiency of up to $30 \%$ making possible the detection of single photons, and its wide dynamic range over 6 orders of magnitudes. As the focal plane of JEM-EUSO will consist of 140 closely packed PDMs, the PDM of EUSO-BALLON has a modular design and is four-side buttable, i.e. its compact array of $6 \times 6$ MAPMT is covering a detection surface of $16.7 \times 16.7 \mathrm{~cm}$, and it is fully contained in a volume of $17 \times 17 \times 20 \mathrm{~cm}^{3}$ with its mechanical support and Front-End electronics included. For the various tasks of photodetection, photodetector polarisation, analog processing, and digital processing, the PDM is composed of the following components and subcomponents:

- 36 MAPMTs organized in,

- 9 Elementary Cell Units (EC-Units), consisting of $2 \times 2$ MAPMTs each,

- the EC ASIC board for their readout (referred to as the EC-ASIC),

- the High-Voltage Power Supply (HVPS)

- the PDM board which includes the FPGA. 


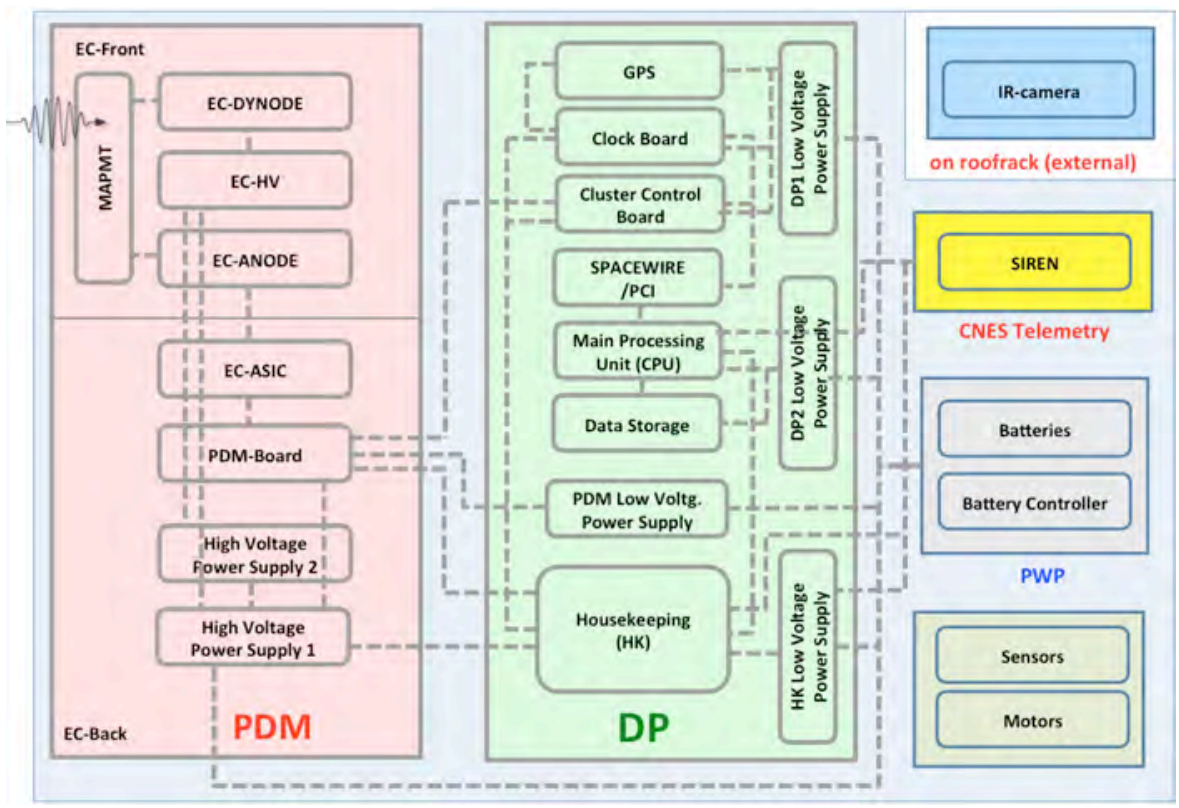

Fig. 2 Functional Block Diagram of the EUSO-Balloon electronics.

The MAPMT's (Hamamatsu R11265-103-M64) have $8 \times 8$ pixels of 2.9 $\times 2.9 \mathrm{~mm}^{2}$ each, and a global footprint of $26.2 \times 26.2 \mathrm{~mm}^{2}$. A UV color glass filter is bonded to the window of the MAPMT with optical glue. The filter (SCHOTT BG3 with anti-reflection coating) transmits UV light in a band between 290 and $430 \mathrm{~nm}$. The Hamamatsu MAPMTs need 14 different high voltages, the highest potential being delivered to the photocathode, the others to the 12 dynodes and the grid. During the flight the photocathode voltage was set to $950 \mathrm{~V}$ which corresponds to a MAPMT gain of roughly $10^{6}$.

The EC-Units are fixed individually to the PDM mechanical frame. At the rear of the MAPMT array, 6 EC-ASIC boards are stacked in parallel inside a mechanical frame with their plane perpendicular to the photocathodes' surfaces. Behind the EC-ASIC stack, the PDM board is fixed with its plane in parallel with the focal surface plane. The HVPS comes in four boxes out of which three are allocated to the nine independent generators and the forth one is dedicated to the control of the switches. The whole EC-Unit block is potted to prevent high voltage sparking at the low-pressure conditions prevailing in the stratosphere.

The EC ASIC is the Front-End electronics performing the MAPMT fulltime analog readout, detecting individual photons in each channel in order to count them per unit of time called the Gate Time Unit (1 GTU $=2.5 \mu \mathrm{s}$ ), performing the analog to digital conversion. The core of the EC-ASIC is the ASIC called SPACIROC V1 (Spatial Photomultiplier Array Counting and Integrating Readout Chip, see [21]), capable of processing in parallel all the 


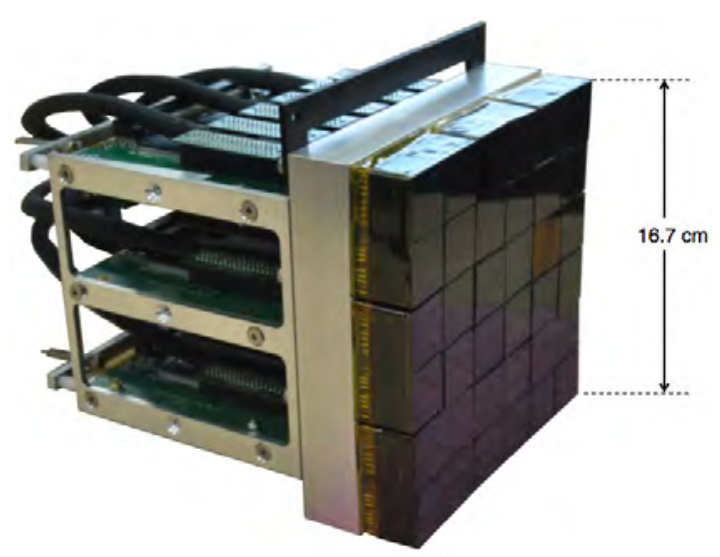

Fig. 3 The Photo-Detector Module (PDM): the MAPMTs are covered with UV filters; four MAPMT's form an Elementary Cell (EC). The PDM includes 9 ECs, 6 EC-boards and a PDM board.

anodes of a single MAPMT. For EUSO-Balloon, the two pulses separation was set at $30 \mathrm{~ns}$ which limited the maximum number of counts per channel to 35 per GTU. In parallel to the 64 photoelectron counting channels, a signal integration per GTU on the anode current sum of groups of 8 adjacent pixels is done so as to correct for counting non-linearity and saturation. Each double sided EC-ASIC board hosts 6 ASIC, three per face.

The High-Voltage Power Supply (HVPS) can operate the MAPMT of an EC-Unit either in the standard photoelectron counting mode with high gain, or in reduced downgraded gain modes in order to face bursts of intense light pulses extending over milliseconds or even more. The HVPS consists of a miniaturized Cockroft-Walton (CW) generator [22] which delivers the 14 high voltages, a switch system used to reduce the photocathode voltage to lower the MAPMT gain, and the logic system to manage the interfaces with the other elements of the electronic chain. The HVPS boxes are required to be potted. The advantage of the $\mathrm{CW}$ generator is its negligible power consumption - an appreciable feature when working from onboard batteries.

The PDM board handles the interfaces between the various boards in the PDM and the Data Processor (6 EC-ASICs, HVPS in the PDM; the HK and CCB from the DP system - see below). Its core is an industrial Virtex6 XC6VLX240T FPGA. Besides managing the configuration, the PDM board governs the the data stream from the 36 ASICs by means of the 6 EC-ASIC boards. The FPGA has to absorb a data-volume of 1 GBytes per second from the EC-Units, transmitting a part of the data to the Central Cluster Board (CCB) of the DP. During the 2014 flight, the order for data transmission was directly sent by the CCB, based on a CPU request. The various parameters of the PDM's performance are treated in detail by a number of articles: photoelectron counting rate measurements [23]; sensitivity and detection performances [24]; absolute calibration [25]. 


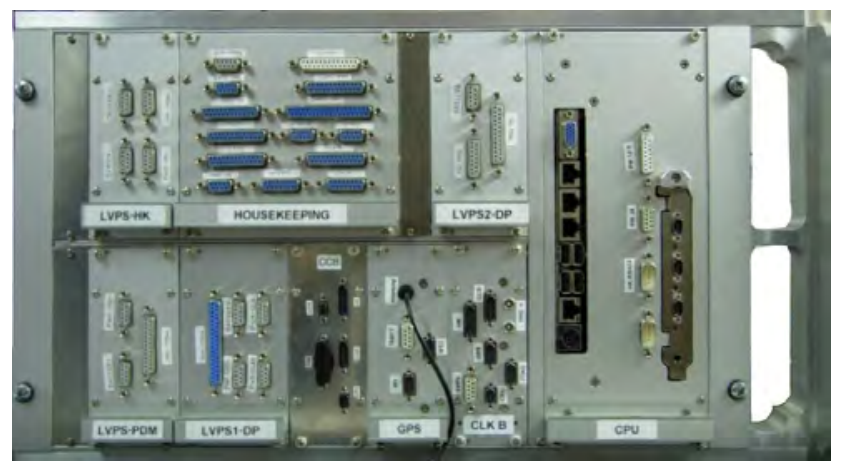

Fig. 4 The Data Processor (DP) collects and processes the data from the PDM. It handles their on-board storage, and sends a subset to the telemetry system. The DP also includes the housekeeping system. The various sub-assemblies are contained in an aluminum crate to assure thermal contact with the radiator.

\subsection{The Data Processor (DP)}

The different sub-assemblies of the DP (Fig. 4) collect the PDM data, process them (trigger, time and position-tagging), handle their on-board storage, and send a subset to the telemetry system. The DP also includes the housekeeping system. The Digital Processor (DP) is composed of

- Control Cluster Board (CCB),

- CPU,

- Data storage (DST),

- Housekeeping system (HK),

- Clock Board (CLKB),

- GPS receiver (GPSR),

- Data Processor Power Supply (DP-LVPS).

The CCB, is developed around an FPGA Xilinx Virtex-4 FX-60, it collects the data from the PDM board, processes and classifies the received data, and performs a second level trigger filtering. The CLKB host the interface with the GPS receiver, it tags the events with their arrival time (UTC) and payload position (both provided by GPSR). It also measures the up-time and dead-time of the instrument and provides signals for time synchronization of the event. Aside from those selected by the two trigger levels, events can be acquired on a signal generated by the CLK-Board. The CLK-Board can send this fake trigger signal on a CPU command. In addition to these two main acquisition modes, two trigger modes linked to 1 Pulse Per Second (1 PPS) signal provided by the GPS receiver are implemented. Both of them are optimized in order to synchronize the acquisition with the light emission from calibrated light sources, a laser or a Xenon flasher, installed on the helicopter flying inside the instrument field of view. Most of the functions of the CLKB are implemented in a FPGA Xilinx Virtex-5 XC5VLX50T. 
The CPU, based on Atom N270 1.6 GHz processor, collects data from the CCB and CLKB through two (200 Mbits/sec) SpaceWire links. It manages the Mass Memory for data storage and handles the interface with the telecommand/telemetry system. One acquired event represents roughly $330 \mathrm{kB}$ of data. Since only a limited data rate can be transmitted to the ground through CNES' new NOSYCA telemetry system, all data are stored on board. The mass storage is composed of two Solid-State Drives (SSD), each one with 512 GB capacity operating in fault-tolerant mode RAID 1 disks (Redundant Array of Independent Disks). The House-Keeping system collects telemetry from several sub-systems of the instrument in slow control mode. It is responsible for monitoring voltages and currents of the Low Voltage Power Supply (LVPS), has a serial bus to convey telemetry and telecommands through the CPU interface and to other sub-systems. The HK is implemented around an off-the-shelf micro-controller board (Arduino Mega 2560), combined with 5 custom-made protocol interface boards to pre-process the various signals. A detailed description of the DP and its performance are given in [26].

\subsection{The optics}

The optical bench contains two Fresnel lenses made from $8 \mathrm{~mm}$ thick PMMA (UV transmitting polymethyl-methacrylate) with a front surface of $100 \times$ $100 \mathrm{~cm}$ each. The EUSO-Balloon optics has been designed to resemble the JEM-EUSO optics: it is dimensioned to produce background rates per pixel comparable to the one anticipated for JEM-EUSO (i.e. roughly $2 \pm 1$ photoelectrons per pixel in a $2.5 \mu \mathrm{sec}$ frame). A ray tracing diagram of the optical bench is shown in Fig. 5: L1 and L3 are aspherical Fresnel Lenses with focal lengths of $258.56 \mathrm{~cm}$ and $60.02 \mathrm{~cm}$, respectively (focal lengths are reference values only, single lenses are not producing stigmatic images). The position of L1 can be adjusted along the optical (Z) axis within the optical bench, the focal distance of the PDM is adjusted by a translation stage in the instrument boot. Together with the $15 \times 15 \mathrm{~cm}$ focal plane detector (PDM, see above) the optics provides a field of view of $\pm 5.5^{\circ}$. A detailed description of the design and manufacturing of the balloon optics is given in [27] and [28]. The measurements of the performance of the fully integrated optical bench (global optical efficiency and point spread function) were performed at IRAP Toulouse and are reported in [29].

\subsection{The Infrared Camera (IRcam)}

In order to monitor the cloud covers and particularly their height, the coaligned IRcam (Fig. 6) observes the field of view of the main instrument. The general design of the IRcam is similar to the one used on JEM-EUSO [30]. The camera provides images with a resolution of $640 \mathrm{x} 480$ pixels, in two wavelength bands centered at $10.8 \mu$ and $12 \mu$ (medium infrared), thanks to a ULIS UL 


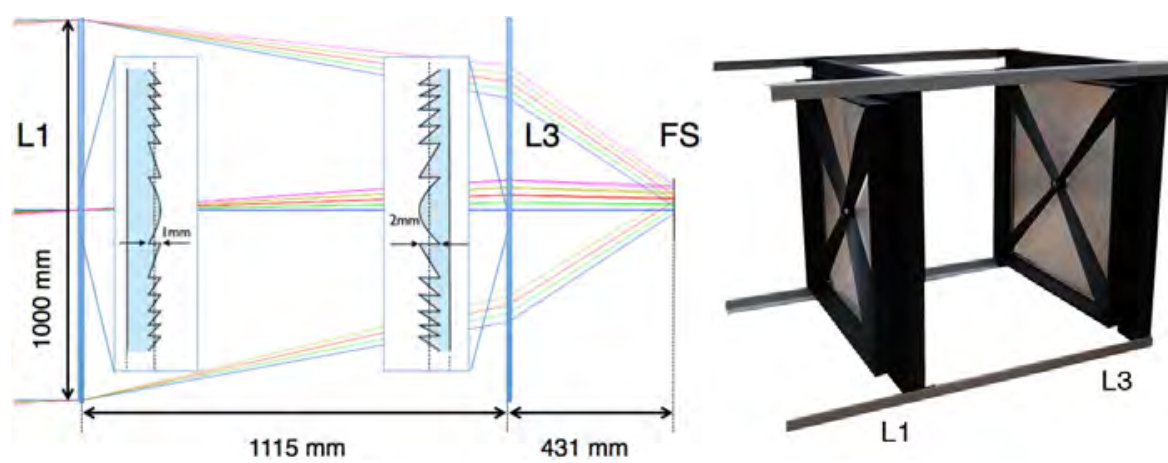

Fig. 5 left: raytracing diagram for the EUSO-Balloon optics with the Fresnel Lenses L1 and L3, and the focal surface FS; the incident rays are at off-axis angles ranging from $0^{\circ}$ (blue) to $1^{\circ}$ (green), $2^{\circ}$ (red) $3^{\circ}$ (yellow) and $4^{\circ}$ (purple); inserts show partial sectional views of L1 and L3; right: the Fresnel lenses L1 and L3 (8 mm thick PMMA, surface of $1 \times 1 \mathrm{~m})$ mounted onto their fiberglass frames and spiders, and held at a distance of $1.11 \mathrm{~m}$ by an optical "sled".

04171 microbolometer and two filters with $0.85 \mu \mathrm{m}$ bandwidth, centered on the above wavelengths. The data from the IRcam, along with auxiliary data (temperature, pressure and humidity), are stored in a RAID1 configuration of 2 SSD of 32 GB of capacity. The entire stand-alone IRcam system, including $\mathrm{CPU}$ and batteries, is housed in a rugged aluminium box $(0.4 \times 0.4 \times 0.4 \mathrm{~m})$ on the outside of the optical bench (see Fig. 1). A detailed description of the IRcam is presented in [31], its performance is detailed in [32].

\subsection{The gondola}

The particular configuration of the nadir pointing instrument allowed to design a simple telescope structure serving simultaneously as a ballon gondola. The structure consists of two main modules, the optical bench and the instrument booth (see Fig. 1), both built from $10 \mathrm{~mm}$ Fibrelam aerospace panels assembled by Fribrolux L profiles. Fibrelam panels are manufactured from honeycomb that is bonded between composite facing skins; they are light-weight, structurally sound and exceptionally stiff. The dimensions of the fibrelam-telescope itself are $1.21 \times 1.21 \times 2.90 \mathrm{~m}$, the size of the flight-ready gondola is $2.58 \times$ $2.58 \times 3.7 \mathrm{~m}$, including "crash-rings" and floaters. The overall launch mass of the integrated instrument is $467 \mathrm{~kg}$.

Inside the self-contained, watertight instrument booth, all electronic equipment is mounted on a system of aluminum "shelves" that provides a thermal link to the $1.2 \times 1.2 \mathrm{~m}$ aluminum backplate (also called radiator). As the flight chain and harness interface to this backplate, it gets almost all the mechanical efforts at the opening shock of the parachutes. The entire gondola has been designed to withstand accelerations of up to $15 \mathrm{~g}$ along the Z-axis, and $5 \mathrm{~g}$ in transverse $(\mathrm{X}, \mathrm{Y})$ directions. Besides its role as a structural element, the role 

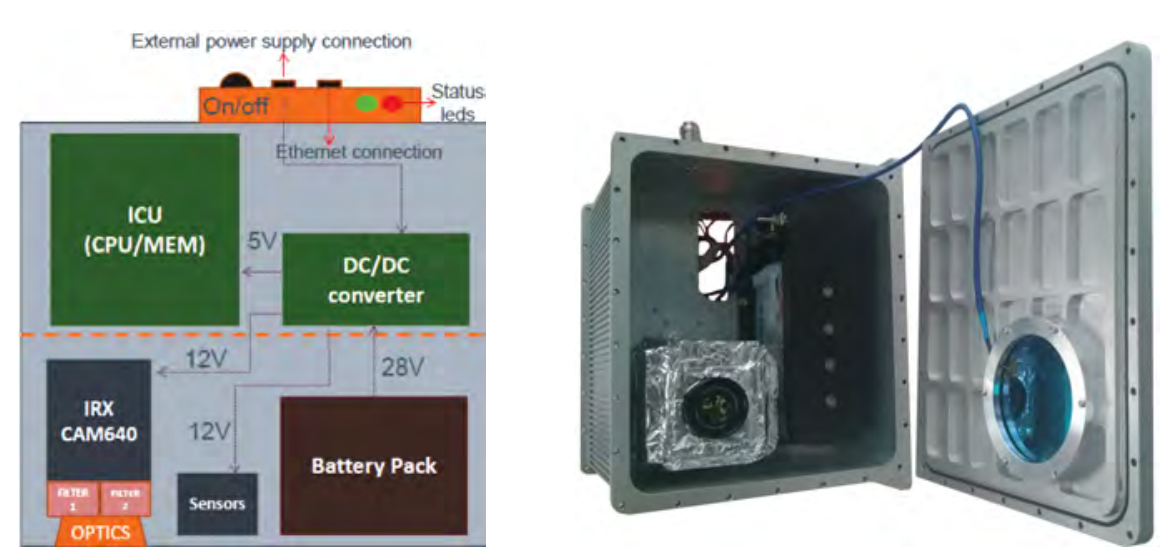

Fig. 6 left: block diagram of the stand-alone IRcam system; right: the rugged watertight aluminium housing $(0.4 \times 0.4 \times 0.4 \mathrm{~m})$ of the IRcam with the IRX CAM640 in its lower left side.

of the radiator is to evacuate the excess energy generated by the electronics towards outer space.

A thermo-mechanical analysis of the instrument was performed to ensure the operation of the various electronic sub-systems in near vacuum conditions within their admissible range $\left(-20^{\circ} \mathrm{C}\right.$ to $\left.30^{\circ} \mathrm{C}\right)$, and to check the mechanical behavior and integrity of the telescope structure in extreme "hot" and "cold" flight scenarios.

The two Fresnel lenses (see above) and their mechanical supports are fixed within the optical bench so that the focal surface coincides with the photocathodes of the Photo-Detector Module. While the rear Fresnel lens (L3) is fixed, closing the watertight instrument booth, the front lens (L1) can be adjusted along the optical $(\mathrm{Z})$ axis; the PDM is mounted on a translation stage, allowing to adjust the distance between PDM and rear lens (L3) .

Instead of "classic" crash-pads made from layered cardboard-honeycomb, $E U S O$-Balloon is using an ensemble of aluminium "crash-rings", designed to absorb the kinetic energy on ground impact through inelastic distortion (Fig $1)$. Due to the coincidence of a science requirement and a safety constraint, EUSO-Balloon has deliberately been designed to protect all sensitive equipment in the event of a water-landing.

In EUSO-Balloon, a number of independent features (Fig. 7) maximize the chances for recovering all sensitive equipment in working condition: 1) To minimize damage to the payload and warrant the integrity of the instrument booth at splashdown, efficient deceleration is achieved by using the instruments optical baffle as a "deceleration-cylinder"; the pressure of the air-cushion in the enclosed volume being passively controlled by calibrated evacuation-holes; 2) the entire instrument booth is held above the waterline by a collar of floaters, whatever the orientation of the gondola in the water may be; 3) during splashdown the instrument booth will be at least immersed partially (and the submersion may become permanent if the floaters are damaged on impact). All 


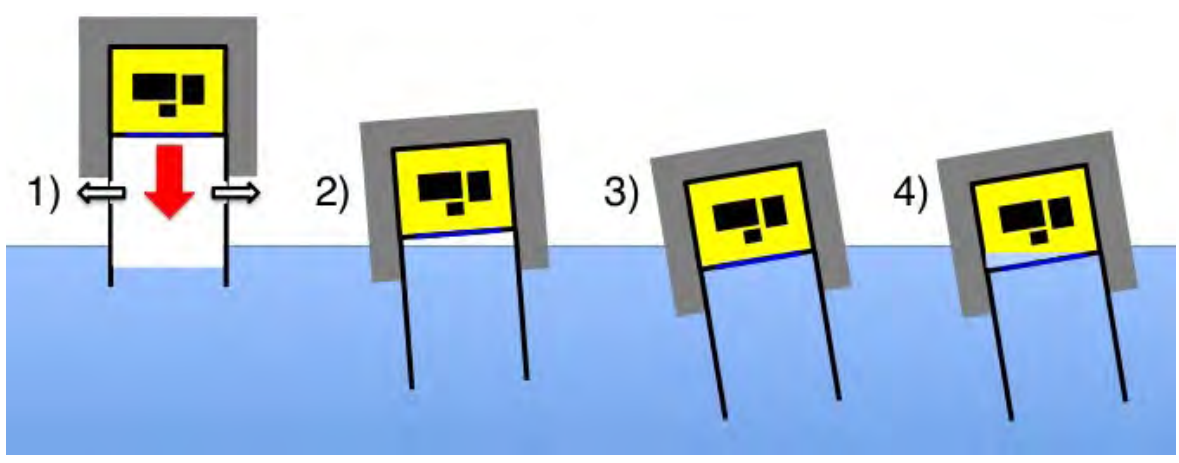

Fig. 7 The features making EUSO-Balloon survive a water-landing: 1) "decelerationcylinder", 2) collar of floaters, 3) watertight instrument booth using the third Fresnel lens (L3) as a porthole, 4) electronics is mounted on a "dry-shelf" above eventual capillary water - see text.

electronics are therefore hosted within the instrument booth which is built as a watertight capsule, using the third Fresnel lens (L3) as a porthole; 4) all sensitive equipment is mounted on a "dry-shelf" with limber-holes, keeping the electronics clear from the inside walls of the instrument booth where eventual capillary water might accumulate.

On its maiden flight in August 2014, EUSO-Balloon not only accomplished its science goals, but it also accidentally splashed down into a tiny solitary lake (barely bigger than two football fields) validating the water-landing capabilities it was designed for in the first place.

\section{The 2014 ballon flight from Timmins, Ontario}

On August 25, 2014 EUSO-Balloon was launched from the Timmins Stratospheric Balloon Base (lat $48.57^{\circ} \mathrm{N}$, lon $81.38 \mathrm{~W}$ ). Thanks to the auxiliary balloon technique routinely used by CNES the entire launch-operations went very smoothly (Fig. 9). The $467 \mathrm{~kg}$ payload was lifted from the airfield by a $400,000 \mathrm{~m}^{3}$ Zodiac balloon at 0:53 UT, reaching a float altitude of $38300 \mathrm{~m}$ at 3:43 UT. The high-voltages of the PDM had already been switched on at 2:50 UT, at an altitude of $32 \mathrm{~km}$, when the balloon was still on its ascent. Telemetry data rapidly indicated that all systems were operating flawlessly, and soon the city-lights of Timmins came into the field of view at the saturation level, confirming that the PDM was in good health and was taking UV images. During more than five hours of operation at float, a total of 258,592 datapackets, corresponding to roughly 33 million frames (GTU's) were recorded and written onto the two redundant hard-drives onboard. With the NOSYCA (S-band) telemetry system featuring a data rate of $\leq 1,75 \mathrm{Mb} / \mathrm{s}$, about one million frames (GTU's) were transmitted to the ground during the flight. The largest part of the science harvest, however, resided on the two hard-drives onboard, and therefore relied upon a safe landing and recovery. 


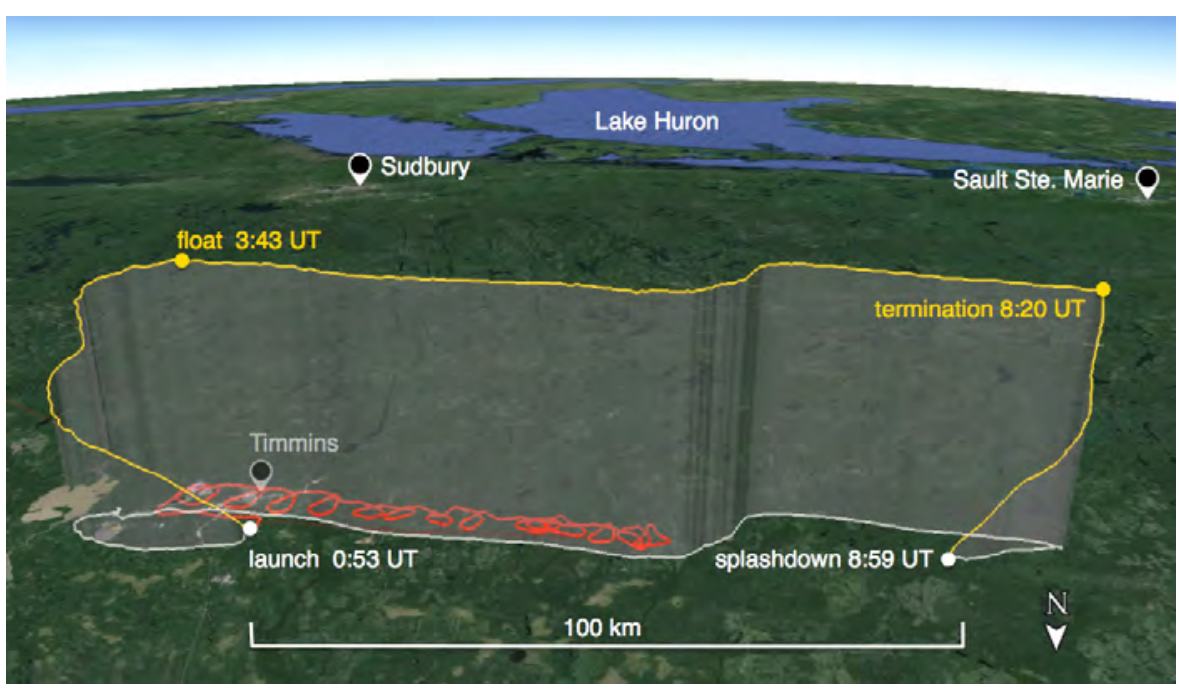

Fig. 8 The flight-track of EUSO-Balloon on August 25, 2014 (yellow) - float altitude was $38 \mathrm{~km}$. The helicopter carrying the UV laser and two UV flashers followed the balloon for over two hours at an altitude of $3000 \mathrm{~m}$ (red).

The moonless night of August 24/25 provided optimal conditions for the study of the UV background: a variety of ground covers have been overflown - including different types of soil and vegetation, wetlands, open water, urban and industrial areas, and various types of clouds. As it seemed unlikely to detect cosmic-ray induced air showers during this first short balloon flight, a pulsed UV laser and two UV flashers (LED and Xe) were shot into the field of view from a helicopter to simulate tracks of UV fluorescence photons, providing a means for the absolute calibration of the instrument [12]. The helicopter followed the balloon for over two hours, flying circles along the flight track at an altitude of $3000 \mathrm{~m}$.

Towards the end of the astronomical night at 8:20 UT, the flight was terminated (separation between the balloon and the instrument which descends on a parachute) about $100 \mathrm{~km}$ to the west of Timmins. Despite a descent path guaranteeing one of the "driest" landing zones along the flight track, EUSO-Balloon and the entire flight train splashed down in a small solitary lake at 8:59 UT. An adventurous recovery was performed by a crew of trappers, EUSO members, and with the help of a helicopter. Thanks to its inborn design for water-landings, the entire instrument was undamaged, both lenses were intact, the electronics, IRcam, and the RAID disks had not suffered any water damage and were fully operational.

\subsection{Summary of the first results}

As the present article focuses on the description of the instrument, the results of the Timmins flight are merely summarized here. For a general overview of 

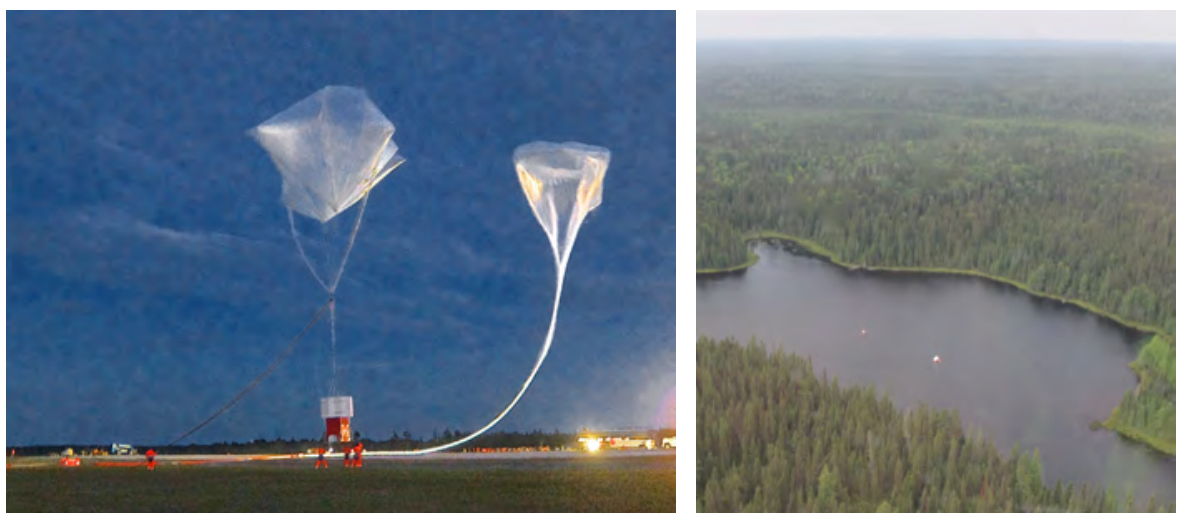

Fig. 9 left: Timmins (Ontario, Canada) Stratospheric Balloon Base, August 25, 2014, 0:53 UT : the perfect launch of EUSO-Balloon by the balloon division of the French Space Agency CNES, the auxiliary balloons (above the payload in the picture) warrant a smooth launch even in case of moderate surface winds. right: the instrument floating in the middle of "Lake Euso" after splashing down at 8:59 UT. All systems survived the impact and more than eight hours in the water thanks to a dedicated "water-landing" design.

the preliminary results, the reader is referred to [7]. A first estimate of the UV background in a clear atmosphere - and with the presence of clouds - has been performed by [9]. By comparing UV and IR images the authors reveal a strong dependence of the upward UV radiance on the atmospheric conditions. Close to 300 laser-shots produced during the helicopter underflight were detected by processing the complete flight data set with algorithm foreseen for the $1^{\text {st }}$ level trigger of JEM-EUSO [11]. The detailed analysis of these laser-shots (determining the Shower Detector Plane and the time projection of the track onto this plane) allowed to reconstruct the laser directions to within a few degrees in 3D [8]. The absolute calibration of the entire EUSO-Balloon system through the airborne light sources (laser, flashers) has been performed by [12]. Detailed simulations of the full instrument (including detection, efficiencies atmospheric absorption etc.) have been performed by [34] to reconstruct the shower profile, deduce the energy and direction resolution, and evaluate the performance of the trigger algoithm. An analysis of the flight data within the Offline framework, and comparing them with simulations, is presented in [13]. Images from the IRcam were used to retrieve the cloud coverage and the Cloud Top Height during times the UV flashers were operated, with the goal to infer the UV optical depth when the atmosphere was not clear [14]. An accurate method for extracting Cloud Top Heights via vertical profiles predicted by the WRF model is developed in [15]. The reliability of this model for the modeling of atmospheric properties has been tested by [16]. Finally, the sensitivity of the instrument to coherent fluctuations of the UV background is investigated over large areas, on timescales ranging from a few microseconds to a few seconds, with the goal to search for significant variations [10]. 


\section{Conclusions and Perspectives}

With its EUSO-Balloon flight in 2014, our collaboration has made a giant step towards demonstrating the key technologies and methods featured in JEM-EUSO. An ample part of the technological objectives ("A" objectives, see section 2) have been achieved during this flight, and the UV background was studied from a variety of ground covers ("B" objectives). The detection of hundreds of laser-shots produced during the helicopter underflight has already partly fulfilled the "C" objectives, namely the detection of (simulated) Extensive Air Showers from above.

In order to achieve the ultimate objective ("C") of EUSO-Balloon, i.e. the first observation of UHECR induced Extensive Air Showers, the collaboration is planing two further balloon flights [33].

A first short flight will be carried out possibly as early as June 2016 with a launch from the CNES balloon-base in Aire-sur-l'Adour (France). The main goal is to test both an improved PDM and an achromatic optical bench, measure background above the Atlantic Ocean as well as simulated EASs (laser/flashers flown on an airplane), and validate strategies for the recovery of hardware and data in a planned splashdown at high sea.

Even tough the performance of the instrument was adequate for the objectives of the Timmins flight, a number of issues need to be ameliorated. The most important improvements for the PDM concern the FPGA, the ASIC and a simplification of the HV electrical architecture. The new firmware and resource allocation implemented in a more performant FPGA will handle a first level trigger, allowing for the detection on-board of EASs (and incidentally dramatically reduce the data volume to be transmitted to the ground). The new ASIC (SPACIROC V3) will enhance the double pulse separation capability from $30 \mathrm{~ns}$ to $10 \mathrm{~ns}$ resolution, extending the dynamic range for photoelectron counting by a factor 3 , from 35 counts per GTU to 100 counts per GTU. A simplified electrical architecture of the HV will permit to run the MAPMTs at $-1100 \mathrm{~V}$ (instead of the $-950 \mathrm{~V}$ for the Timmins flight), resulting in a gain of $G=10^{6}$, amply reducing electronic noise and facilitate separation from single photoelectron signal. The main improvement of the optics is the addition of a flat diffractive lens (L2) between L1 and L3 which will correct the chromatic aberration of the system, and obtain a small RMS spot size of $\sim 3 \mathrm{~mm}$ (pixel size of the PDM).

The second long or ultra-long duration balloon (ULDB) flight shall achieve our ultimate goal, i.e. measuring a significant sample of UHECR induced tracks of UV light "from above". The flight could take place in spring 2017, most likely with a launch from the recently inaugurated base at Wanaka (NZ) by the Columbia Scientific Balloon Facility (CSBF/NASA). For this flight, the instrument will be supplemented with solar panels and a power management system guaranteeing virtually unlimited continuous operation. The trigger efficiency has been determined as a function of energy by [35], deriving the number of events to be expected in the range $10^{17}$ to $10^{19} \mathrm{eV}$, demonstrating that EUSO-Balloon is able to detect fluorescence produced by EASs from 
above. The first observation of UHECR induced Extensive Air Showers from near space is an important breakthrough, paving the way for JEM-EUSO, and for any future space-based UHECR observatory.

Acknowledgment: The authors acknowledge strong support from the French Space Agency CNES who provided - besides funding - the leadership that made this achievement possible in a very short time. We are deeply indebted to the balloon division of CNES for a perfect launch, smooth flight operation and flawless telemetry. The Canadian Space Agency has provided outstanding facilities at the Timmins Stratospheric Balloon Base, and a quick and careful recovery of the instrument. We would like to thank our laboratories and the entire JEM-EUSO collaboration for their strong and undivided support all along this project.

This work was partially supported by Basic Science Interdisciplinary Research Projects of RIKEN and JSPS KAKENHI Grant (22340063, 23340081, and 24244042), by the Italian Ministry of Foreign Affairs, General Direction for the Cultural Promotion and Cooperation, by the 'Helmholtz Alliance for Astroparticle Physics HAP' funded by the Initiative and Networking Fund of the Helmholtz Association, Germany, and by Slovak Academy of Sciences MVTS JEM-EUSO as well as VEGA grant agency project 2/0076/13. The Spanish consortium involved in the JEM-EUSO Space Mission is funded by MICINN under projects AYA2009- 06037-E/ESP, AYA-ESP 2010-19082, AYA2011-29489-C03- 01, AYA2012-39115C03-01, CSD2009-00064 (Consolider MULTIDARK) and by Comunidad de Madrid (CAM) under project S2009/ESP-1496.

\section{References}

1. Adams, J.H. et al. (The JEM-EUSO Collaboration), The JEM-EUSO mission, this volume.

2. Adams, J.H. et al. (The JEM-EUSO Collaboration), Ultra High Energy Cosmic Rays studies with the JEM-EUSO mission, this volume.

3. Adams, J.H. et al. (The JEM-EUSO Collaboration), Ultra High Energy Photons and Neutrinos with JEM-EUSO, this volume.

4. Adams, J.H. et al. (The JEM-EUSO Collaboration), An overview of the JEM-EUSO instrument, this volume.

5. The Pierre Auger Collaboration, Phys.Lett. B685:239-246, 2010, Measurement of the energy spectrum of cosmic rays above $10^{18} \mathrm{eV}$ using the Pierre Auger Observatory

6. Fukushima, M., for the Telescope Array Collaboration, proc. ISVHECRI-2014 symposium at CERN, arXiv:1503.06961, 2015, Recent Results from Telescope Array

7. Bertaina, M., et al., Proc. $34^{\text {th }}$ ICRC, 2015, Preliminary results from the EUSO-Balloon flight

8. Eser, J., et al., Proc. $33^{\text {rd }}$ ICRC, 2015, Laser direction reconstruction in EUSO-Balloon experiment

9. Mackovjak, S.,34 $34^{\text {th }}$ et al., Proc. $34^{\text {th }}$ ICRC, 2015, Night time measurement of the UV background by EUSO-Balloon

10. Jung, J., et al., Proc. $34^{\text {th }}$ ICRC, 2015, Search for significant background fluctuations in the EUSO-Balloon data

11. Suino, G., et al., Proc. $34^{\text {th }}$ ICRC, 2015, Tests of JEM-EUSO 1st level trigger using EUSO-Balloon data

12. Adams, J., et al., Proc. $34^{\text {th }}$ ICRC, 2015, The Calibration of EUSO-Balloon using airborne light sources mounted to a Helicopter

13. Panico, B., et al., Proc. $34^{\text {th }}$ ICRC, 2015, Analysis of EUSO-Balloon data with Offline

14. Saez, G., et al., Proc. $34^{\text {th }}$ ICRC, 2015, Cloud Optical Depth obtained from the IR

Camera data and the UV Flashers flying under the EUSO-Balloon (CNES)

15. Merino, A., et al., Proc. $34^{\text {th }}$ ICRC, 2015, Cloud top height estimation from WRF model: application to the infrared camera onboard EUSO-Balloon (CNES)

16. Tabone, I., et al., Proc. $34^{t h}$ ICRC, 2015, The WRF model contribution to the atmospheric conditions estimation during the EUSO-Balloon experiment 
17. Catalano, O., et al.: The atmospheric nightglow in the $300-400 \mathrm{~nm}$ wavelength: Results by the balloon-borne experiment BABY. Nucl. Inst. Methods A 480, 547-554 (2002)

18. Barbier, M., et al.: NIGHTGLOW: An instrument to measure the Earth's nighttime ultraviolet glow - results from the first engineering flight. Astropart. Phys. 22, 439-449 (2005)

19. Garipov, G.K., et al.: UV radiation from the atmosphere: Results of the MSU Tatiana satellite measurements. Astropart. Phys. 24, 400-408 (2005)

20. Mernik, T., et al., Proc. 33 ${ }^{\text {rd }}$ ICRC, 2013 (ID777), ESAF-Simulation of the EUSOBalloon

21. Ahmad, S., et al., Proc. $33^{r d}$ ICRC, 2013 (ID1089), Performance of the SPACIROC front-end ASIC for JEM-EUSO

22. Blaksley, C., et al., Proc. $34^{\text {th }}$ ICRC, 2015, A Cockcroft-Walton High-Voltage Power Supply for the EUSO Instruments

23. Rabanal, J., et al., Proc. $34^{t h}$ ICRC, 2015, Photoelectron counting rate measurements in the UV camera during the EUSO-BALLOON night flight

24. Dagoret, S., et al., Proc. $34^{\text {th }}$ ICRC, 2015, Determination of the sensitivity and detection performances of the UV camera pixels of the EUSO-BALLOON instrument

25. Moretto, C., et al., Proc. $34^{t h}$ ICRC, 2015, Absolute calibration of the photon detector module of the EUSO-Balloon experiment and improvements for future missions

26. Osteria, G. et al., Proc. $34^{\text {th }}$ ICRC, 2015, The Data Processor System of EUSO-Balloon: in flight performance

27. Takizawa, Y., Zuccaro, A., Proc. $33^{\text {rd }}$ ICRC, 2013 (ID832), The TA-EUSO and EUSOBalloon optics designs

28. Takizawa, Y., et al., Proc. $33^{\text {rd }}$ ICRC, 2013 (ID1040), Manufacturing of the TA-EUSO and the EUSO-Balloon lenses

29. Catalano, C. et al., Proc. $34^{\text {th }}$ ICRC, 2015, Performance of the EUSO-Balloon optics

30. Morales et al., J.A., Proc. 33 ${ }^{\text {rd }}$ ICRC, 2013 (ID514), An End to End Simulation code for the IR-Camera of the JEM-EUSO Space Observatory

31. Rodriguez Firas, M.D., et al., Proc. $34^{\text {th }}$ ICRC, 2015, The Spanish Infrared Camera onboard the EUSO-Balloon (CNES) flight on August 24, 2015

32. Fernandez, J., et al., Proc. $34^{\text {th }}$ ICRC, 2015, Performances of the Spanish Infrared Camera onboard the EUSO-Balloon (CNES) flight on August 24, 2014

33. Wiencke, L., et al., Proc. $34^{\text {th }}$ ICRC, 2015,EUSO-Balloon mission to record extensive air showers from near space

34. Fenu, F., et al., Proc. $34^{\text {th }}$ ICRC, 2015, The Simulation of cosmic rays in EUSO-Balloon: performance in the direction and energy reconstruction

35. Bacholle, S., et al., Proc. $34^{\text {th }}$ ICRC, 2015,EUSO-Balloon trigger efficiency in preparation of a long duration flight 


\title{
The JEM-EUSO Collaboration
}

\author{
J.H. Adams Jr. ${ }^{m d}$, S. Ahmad ${ }^{b b}$, J.-N. Albert ${ }^{b a}$, D. Allard ${ }^{b c}$, L. Anchordoqui ${ }^{m f}$, V. Andreev ${ }^{m e}$,

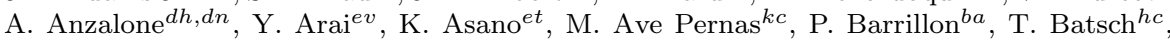

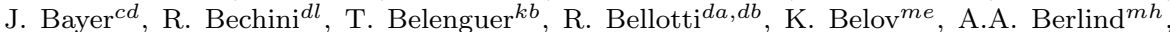 \\ M. Bertaina ${ }^{d k, d l}$, P.L. Biermann ${ }^{c b}$, S. Biktemerova ${ }^{i a}$, C. Blaksley $^{b c}$, N. Blanc ${ }^{l a}$, J. Błẹcki $^{h d}$, \\ S. Blin-Bondil ${ }^{b b}$, J. Blümer ${ }^{c b}$, P. Bobik ${ }^{j a}$, M. Bogomilov ${ }^{a a}$, M. Bonamente ${ }^{m d}$, M.S. Briggs $^{m d}$,

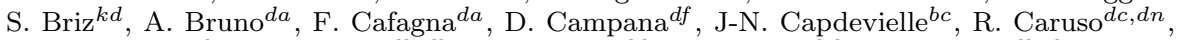

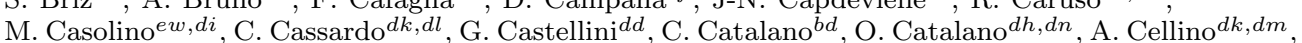 \\ M. Chikawa ${ }^{e d}$, M.J. Christl ${ }^{m g}$, D. Cline ${ }^{m e}$, V. Connaughton ${ }^{m d}$, L. Conti ${ }^{d o}$, G. Cordero ${ }^{g a}$, \\ H.J. Crawford ${ }^{m a}$, R. Cremonini ${ }^{d l}$, S. Csorna ${ }^{m h}$, S. Dagoret-Campagne ${ }^{b a}$, A.J. de Castro ${ }^{k d}$, \\ C. De Donato ${ }^{d i}$, C. de la Taille ${ }^{b b}$, C. De Santis ${ }^{d i, d j}$, L. del Peral ${ }^{k c}$, A. Dell'Oro ${ }^{d k, d m}$ N. De \\ Simone $^{d i}$, M. Di Martino ${ }^{d k, d m}$, G. Distratis ${ }^{c d}$, F. Dulucq ${ }^{b b}$, M. Dupieux ${ }^{b d}$, A. Ebersoldt ${ }^{c b}$, \\ T. Ebisuzakie ${ }^{e w}$, R. Engel ${ }^{c b}$, S. Falk ${ }^{c b}$, K. Fang ${ }^{m b}$, F. Fenu ${ }^{c d}$, I. Fernández-Gómez ${ }^{k d}$, S. Ferrarese ${ }^{d k, d l}$, \\ D. Finco ${ }^{d o}$, M. Flamini ${ }^{d o}$, C. Fornaro ${ }^{d o}$, A. Franceschi ${ }^{d e}$, J. Fujimoto ${ }^{e v}$, M. Fukushima $^{e g}$, \\ P. Galeotti ${ }^{d k, d l}$, G. Garipov ${ }^{i c}$, J. Geary ${ }^{m d}$, G. Gelmini ${ }^{m e}$, G. Giraudo ${ }^{d k}$, M. Gonchar ${ }^{i a}$, \\ C. González Alvarado ${ }^{k b}$, P. Gorodetzky ${ }^{b c}$, F. Guarino ${ }^{d f}, d g$, A. Guzmán ${ }^{c d}$, Y. Hachisu ${ }^{e w}$, \\ B. Harlov ${ }^{i b}$, A. Haungs ${ }^{c b}$, J. Hernández Carretero ${ }^{k c}$, K. Higashide $^{e r, e w}$, D. Ikeda ${ }^{e g}$, H. Ikeda $^{e p}$,

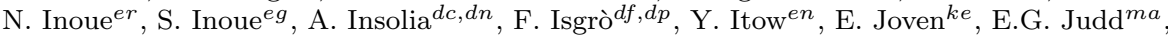 \\ A. Jung ${ }^{f b}$, F. Kajino ${ }^{e i}$, T. Kajino ${ }^{e l}$, I. Kaneko ${ }^{e w}$, Y. Karadzhov ${ }^{a a}$, J. Karczmarczyk ${ }^{h c}$, \\ M. Karus ${ }^{c b}$, K. Katahira ${ }^{e w}$, K. Kawai ${ }^{e w}$, Y. Kawasaki ${ }^{e w}$, B. Keilhauer ${ }^{c b}$, B.A. Khrenov ${ }^{i c}$, \\ Jeong-Sook Kim ${ }^{f a}$, Soon-Wook Kim ${ }^{f a}$, Sug-Whan Kim ${ }^{f d}$, M. Kleifges ${ }^{c b}$, P.A. Klimov ${ }^{i c}$, \\ D. Kolev ${ }^{a a}$, I. Kreykenbohm ${ }^{c a}$, K. Kudela ${ }^{j a}$, Y. Kurihara ${ }^{e v}$, A. Kusenko ${ }^{m e}$, E. Kuznetsov ${ }^{m d}$,

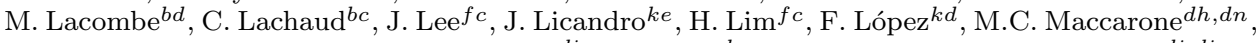

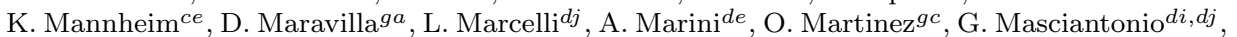 \\ K. Mase ${ }^{e a}$, R. Matev ${ }^{a a}$, G. Medina-Tanco ${ }^{g a}$, T. Mernik ${ }^{c d}$, H. Miyamoto ${ }^{b a}$, Y. Miyazaki $^{e c}$, \\ Y. Mizumoto $^{e l}$, G. Modestino ${ }^{d e}$, A. Monaco ${ }^{d a}, d b$, D. Monnier-Ragaigne ${ }^{b a}$, J.A. Morales de \\ los Ríos ${ }^{k a, k c}$, C. Moretto ${ }^{b a}$, V.S. Morozenko ${ }^{i c}$, B. Mot ${ }^{b d}$, T. Murakami ${ }^{e f}$, M. Nagano ${ }^{e c}$ \\ M. Nagata ${ }^{e h}$, S. Nagatakiek ${ }^{e k}$ T. Nakamura ${ }^{e j}$, T. Napolitano ${ }^{d e}$, D. Naumov ${ }^{i a}$, R. Nava $^{g a}$,

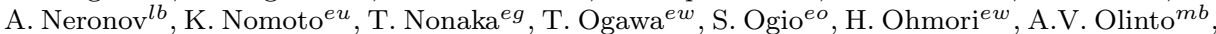 \\ P. Orleański ${ }^{h d}$, G. Osteria ${ }^{d f}$, M.I. Panasyuk ${ }^{i c}$, E. Parizot ${ }^{b c}$, I.H. Park ${ }^{f c}$, H.W. Park ${ }^{f c}$, \\ B. Pastircak ${ }^{j a}$, T. Patzak ${ }^{b c}$, T. Paul ${ }^{m f}$, C. Pennypacker ${ }^{m a}$, S. Perez Cano ${ }^{k c}$, T. Peter ${ }^{l c}$ \\ P. Picozza ${ }^{d i, d j, e w}$, T. Pierog ${ }^{c b}$, L.W. Piotrowski ${ }^{e w}$, S. Piraino ${ }^{c d, d h}$, Z. Plebaniak ${ }^{h c}$, A. Pollini ${ }^{l a}$, \\ P. Prat ${ }^{b c}$, G. Prévôt ${ }^{b c}$, H. Prieto ${ }^{k c}$, M. Putis ${ }^{j a}$, P. Reardon ${ }^{m d}$, M. Reyes ${ }^{k e}$, M. Riccide, \\ I. Rodríguez ${ }^{k d}$, M.D. Rodríguez Frías ${ }^{k c}$, F. Ronga ${ }^{d e}$, M. Roth $^{c b}$, H. Rothkaehl ${ }^{h d}$, G. Roudil ${ }^{b d}$, \\ I. Rusinov ${ }^{a a}$, M. Rybczyński ${ }^{h a}$, M.D. Sabau ${ }^{k b}$, G. Sáez Cano ${ }^{k c}$, H. Sagawa ${ }^{e g}$, A. Saito ${ }^{e j}$, \\ N. Sakaki ${ }^{c b}$, M. Sakata ${ }^{e i}$, H. Salazar ${ }^{g c}$, S. Sánchez ${ }^{k d}$, A. Santangelo ${ }^{c d}$, L. Santiago Crúz ${ }^{g a}$, \\ M. Sanz Palomino ${ }^{k b}$, O. Saprykin ${ }^{i b}$, F. Sarazin ${ }^{m c}$, H. Sato ${ }^{e i}$, M. Sato ${ }^{e s}$, T. Schanz ${ }^{c d}$, \\ H. Schieler ${ }^{c b}$, V. Scotti ${ }^{d f, d g}$, A. Segreto ${ }^{d h, d n}$, S. Selmane ${ }^{b c}$, D. Semikoz ${ }^{b c}$, M. Serra ${ }^{k e}$, \\ S. Sharakin ${ }^{i c}$, T. Shibata ${ }^{e q}$, H.M. Shimizu ${ }^{e m}$, K. Shinozakiew, T. Shirahama ${ }^{e r}$, G. Siemieniec- \\ Oziębło $^{h b}$, H.H. Silva López ${ }^{g a}$, J. Sledd $^{m g}$, K. Słomińska ${ }^{h d}$, A. Sobey ${ }^{m g}$, T. Sugiyama ${ }^{e m}$ \\ D. Supanitsky ${ }^{g a}$, M. Suzuki ${ }^{e p}$, B. Szabelska ${ }^{h c}, J_{\text {. Szabelski }}{ }^{h c}$, F. Tajima ${ }^{e e}$, N. Tajima ${ }^{e w}$, \\ T. Tajima ${ }^{c c}$, Y. Takahashi ${ }^{e s}$, H. Takami ${ }^{e v}$, M. Takeda ${ }^{e g}$, Y. Takizawa ${ }^{e w}$, C. Tenzer ${ }^{c d}$,

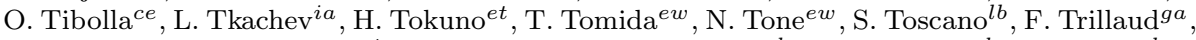 \\ R. Tsenov ${ }^{a a}$, Y. Tsunesada ${ }^{e t}$, K. Tsuno ${ }^{e w}$, T. Tymieniecka ${ }^{h c}$, Y. Uchihori ${ }^{e b}$, M. Unger ${ }^{c b}$, \\ O. Vaduvescu ${ }^{k e}$, J.F. Valdés-Galicia ${ }^{g a}$, P. Vallania ${ }^{d k, d m}$, L. Valore ${ }^{d f, d g}$, G. Vankova ${ }^{a a}$, \\ C. Vigorito ${ }^{d k, d l}$, L. Villaseñor ${ }^{g b}$, P. von Ballmoos ${ }^{b d}$, S. Wada ${ }^{e w}$, J. Watanabe $^{e l}$, S. Watanabe ${ }^{e s}$, \\ J. Watts Jr. ${ }^{m d}$, M. Weber ${ }^{c b}$, T.J. Weiler ${ }^{m h}$, T. Wibig ${ }^{h c}$, L. Wiencke ${ }^{m c}$, M. Wille ${ }^{c a}$, J. Wilms $^{c a}$, \\ Z. Włodarczyk ${ }^{h a}$, T. Yamamoto ${ }^{e i}$, Y. Yamamoto ${ }^{e i}$, J. Yang ${ }^{f b}$, H. Yano ${ }^{e p}$, I.V. Yashin ${ }^{i c}$, \\ D. Yonetoku ${ }^{e f}$, K. Yoshida ${ }^{e i}$, S. Yoshida ${ }^{e a}$, R. Young ${ }^{m g}$, M.Yu. Zotov ${ }^{i c}$, A. Zuccaro Marchiew \\ aa St. Kliment Ohridski University of Sofia, Bulgaria \\ ba LAL, Univ Paris-Sud, CNRS/IN2P3, Orsay, France \\ ${ }^{b b}$ Omega, Ecole Polytechnique, CNRS/IN2P3, Palaiseau, France \\ ${ }^{b c}$ APC, Univ Paris Diderot, CNRS/IN2P3, CEA/Irfu, Obs de Paris, Sorbonne Paris Cité, \\ France
}


${ }^{b d}$ IRAP, Université de Toulouse, CNRS, Toulouse, France

ca ECAP, University of Erlangen-Nuremberg, Germany

$c b$ Karlsruhe Institute of Technology (KIT), Germany

cc Ludwig Maximilian University, Munich, Germany

${ }^{c d}$ Institute for Astronomy and Astrophysics, Kepler Center, University of Tübingen, Germany

ce Institut für Theoretische Physik und Astrophysik, University of Würzburg, Germany

da Istituto Nazionale di Fisica Nucleare - Sezione di Bari, Italy

$d b$ Universita' degli Studi di Bari Aldo Moro and INFN - Sezione di Bari, Italy

dc Dipartimento di Fisica e Astronomia - Universita' di Catania, Italy

$d d$ Consiglio Nazionale delle Ricerche (CNR) - Istituto di Fisica Applicata Nello Carrara, Firenze, Italy

de Istituto Nazionale di Fisica Nucleare - Laboratori Nazionali di Frascati, Italy

$d f$ Istituto Nazionale di Fisica Nucleare - Sezione di Napoli, Italy

$d g$ Universita' di Napoli Federico II - Dipartimento di Scienze Fisiche, Italy

$d h$ INAF - Istituto di Astrofisica Spaziale e Fisica Cosmica di Palermo, Italy

di Istituto Nazionale di Fisica Nucleare - Sezione di Roma Tor Vergata, Italy

dj Universita' di Roma Tor Vergata - Dipartimento di Fisica, Roma, Italy

$d k$ Istituto Nazionale di Fisica Nucleare - Sezione di Torino, Italy

$d l$ Dipartimento di Fisica, Universita' di Torino, Italy

$d m$ Osservatorio Astrofisico di Torino, Istituto Nazionale di Astrofisica, Italy

$d n$ Istituto Nazionale di Fisica Nucleare - Sezione di Catania, Italy

do UTIU, Dipartimento di Ingegneria, Rome, Italy

$d p$ DIETI, Universita' degli Studi di Napoli Federico II, Napoli, Italy

ea Chiba University, Chiba, Japan

${ }^{e b}$ National Institute of Radiological Sciences, Chiba, Japan

ec Fukui University of Technology, Fukui, Japan

ed Kinki University, Higashi-Osaka, Japan

ee Hiroshima University, Hiroshima, Japan

ef Kanazawa University, Kanazawa, Japan

eg Institute for Cosmic Ray Research, University of Tokyo, Kashiwa, Japan

eh Kobe University, Kobe, Japan

ei Konan University, Kobe, Japan

ej Kyoto University, Kyoto, Japan

ek Yukawa Institute, Kyoto University, Kyoto, Japan

el National Astronomical Observatory, Mitaka, Japan

em Nagoya University, Nagoya, Japan

en Solar-Terrestrial Environment Laboratory, Nagoya University, Nagoya, Japan

eo Graduate School of Science, Osaka City University, Japan

${ }^{e p}$ Institute of Space and Astronautical Science/JAXA, Sagamihara, Japan

eq Aoyama Gakuin University, Sagamihara, Japan

er Saitama University, Saitama, Japan

es Hokkaido University, Sapporo, Japan

et Interactive Research Center of Science, Tokyo Institute of Technology, Tokyo, Japan

eu University of Tokyo, Tokyo, Japan

ev High Energy Accelerator Research Organization (KEK), Tsukuba, Japan

ew RIKEN Advanced Science Institute, Wako, Japan

fa Korea Astronomy and Space Science Institute (KASI), Daejeon, Republic of Korea

$f b$ Ewha Womans University, Seoul, Republic of Korea

fc Sungkyunkwan University, Seoul, Republic of Korea

$f d$ Center for Galaxy Evolution Research, Yonsei University, Seoul, Republic of Korea

ga Universidad Nacional Autónoma de México (UNAM), Mexico

${ }^{g b}$ Universidad Michoacana de San Nicolas de Hidalgo (UMSNH), Morelia, Mexico

gc Benemérita Universidad Autónoma de Puebla (BUAP), Mexico

ha Jan Kochanowski University, Institute of Physics, Kielce, Poland

$h b$ Jagiellonian University, Astronomical Observatory, Krakow, Poland

$h c$ National Centre for Nuclear Research, Lodz, Poland

$h d$ Space Research Centre of the Polish Academy of Sciences (CBK), Warsaw, Poland 
ia Joint Institute for Nuclear Research, Dubna, Russia

ib Central Research Institute of Machine Building, TsNIIMash, Korolev, Russia

ic Skobeltsyn Institute of Nuclear Physics, Lomonosov Moscow State University, Russia

ja Institute of Experimental Physics, Kosice, Slovakia

$k a$ Consejo Superior de Investigaciones Científicas (CSIC), Madrid, Spain

${ }^{k b}$ Instituto Nacional de Técnica Aeroespacial (INTA), Madrid, Spain

$k^{k c}$ Universidad de Alcalá (UAH), Madrid, Spain

${ }^{k d}$ Universidad Carlos III de Madrid, Spain

ke Instituto de Astrofísica de Canarias (IAC), Tenerife, Spain

la Swiss Center for Electronics and Microtechnology (CSEM), Neuchâtel, Switzerland

${ }^{l b}$ ISDC Data Centre for Astrophysics, Versoix, Switzerland

${ }^{l c}$ Institute for Atmospheric and Climate Science, ETH Zürich, Switzerland

ma Space Science Laboratory, University of California, Berkeley, USA

$m b$ University of Chicago, USA

$m c$ Colorado School of Mines, Golden, USA

$m d$ University of Alabama in Huntsville, Huntsville, USA

me University of California (UCLA), Los Angeles, USA

$m f$ University of Wisconsin-Milwaukee, Milwaukee, USA

$m g$ NASA - Marshall Space Flight Center, USA

$m h$ Vanderbilt University, Nashville, USA 